SEÇÃO ESPECIAL

\title{
EL NEOLIBERALISMO EN EL MUNDO Y LA PRECARIZACIÓN DEL TRABAJO: UN ESTUDIO HISTÓRICO COMPARADO EN ALEMANIA, ESPAÑA, REINO UNIDO, ITALIA, BRASIL Y MÉXICO
}

\section{Fernanda Barcellos}

\section{Mathiasi}

Doutoranda em Ciências

Sociais pela Universidade

Autônoma de Sinaloa,

México, e Universidade

Federal de Juiz de fora, pela

modalidade de Cotutela. Juiz

de Fora, Minas Gerais Brasil.

fernandabarcellosmathiasi@

gmail.com

\section{Thiago Duarte Pimentel}

Professor Adjunto da

Universidade Federal de Juiz de Fora, Juiz de Fora/MG, Brasil. Doutor em Ciências Sociais pela Universidade Federal de Juiz de Fora, Juiz de Fora/MG, Brasil. E-mail: Thiago.pimentel@ich.ufjf.br

\section{O neoliberalismo no mundo e a precarização do trabalho: um estudo comparado entre Alemanha, Espanha, Reino Unido, Itália, Brasil e México}

\section{RESUMEN}

El objetivo de este artículo científico es comprender y debatir los efectos del discurso neoliberal que llevaron a reformas en las leyes laborales en algunos países del mundo como Alemania, España, Brasil, México, Reino Unido e Italia, desde la década de 1980 hasta los días actuales, en un análisis bibliográfica de artículos científicos que presentan las características del mercado laboral en cada país y las razones que fundamentar un cambio legislativo de menos garantías y derechos a los trabajadores, relacionando así los objetivos legales y los efectos reales en el mercado de trabajo. La pregunta de investigación es: ¿Que tipo de impacto en el mercado laboral ha generado las reformas en las leyes laborales en Alemania, España, Reino Unido, Italia, Brasil y México? ¿Cuáles fueron los resultados de las reformas laborales en estos países en términos de los criterios de considerados por Kallenberg (2009) para la precariedad laboral? Un especial enfoque al caso de la Reforma Laboral de 2012 en México y 2017 en Brasil de los indicadores de precarización del trabajo 
Recebido: abril, 2019

Aceito: agosto, 2019 de la OIT. Particularmente, se vale aquí de fuentes secundarias bibliográficas con estadísticas nacionales y datos históricos para fines de identificar los cambios hechos y la precarización del trabajo.

Palabras-clave: Neoliberalismo; Crisis del Estado de Bienestar Social; Precarización del Trabajo; Reforma Laboral

\section{Resumo}

O objetivo deste artigo científico é compreender e debater os efeitos do discurso neoliberal por meio de reformas nas leis trabalhistas em alguns países do mundo como Alemanha, Espanha, Brasil, México, Reino Unido e Itália, desde a década de 1980 até os dias atuais, em uma análise bibliográfica de artigos científicos que apresentam as características do mercado de trabalho em cada país, e as razões que fundamentaram uma mudança legislativa de menos garantias e direitos aos trabalhadores, assim, relacionando os objetivos legais e os efeitos reais no mercado trabalho. Um especial enfoque ao caso da Reforma Trabalhista de 2012 no México e 2017 no Brasil e dos indicadores de precarização do trabalho da OIT (Organização Internacional do Trabalho). A pregunta de investigacao é: Que tipo de impacto no mercado de trabalho a reforma nas leis trabalhistas geraram na Alemania, España, Reino Unido, Italia, Brasil y México? Quais foram os resultados da reforma trabalhista nestes países segundo os considerados por Kallenberg (2009) para a precariedade laboral? Em particular, utiliza fontes bibliográficas secundárias com estatísticas nacionais e dados históricos com o objetivo de identificar as mudanças realizadas e a precariedade do trabalho.

Palavras-chave: Neoliberalismo; Crise do Estado de Bem-Estar Social; Precarização do Trabalho; Reforma Trabalhista.

\section{Abstract}

Abstract: The purpose of this scientific article is to understand and debate the effects of neoliberal discourse through labor law reforms in some countries of the world such as Germany, Spain, Brazil, Mexico, the United Kingdom and Italy, from the 1980s to the present day, in a bibliographical analysis of scientific articles that present the characteristics of the labor market in each country and the reasons that underlie a legislative change of less guarantees and rights to the workers, thus, relating the legal objectives and the real effects in the labor market. A special focus on the case of the 2012 Labor Reform in Mexico and 2017 in Brazil and the ILO (International Labor Organization) labor precariousness indicators. The research question is: What kind of impact on the labor market has labor law reform generated in Germany, Spain, the United Kingdom, Italy, Brazil and Mexico? What were the results of labor reform in these countries according to those considered by Kallenberg (2009) for labor precariousness? In particular, it uses secondary bibliographic sources with national statistics and historical data to identify changes made and the precariousness of work.

Palavras-chave: Neoliberalism; Social Welfare State Crisis; Labor Precarization; Labor Reform. 


Nuestro
argumento
central es que
dichas reformas
hacen parte de
un movimiento
más amplio de
reestructuración
del capital, en lo
que respecta a la
geopolítica crítica
y la nueva división
internacional
del trabajo, en
las sociedades
contemporáneas,
configurando así
una verdadera
"ola", en que ha
ascendido
la agenda
neoliberal [...]
das que sirvan de guía para la acción². En el ámbito pragmático, una cuestión está en los derechos garantizados en las constituciones del mundo otro hecho es la efectividad de los derechos y otra cuestión es la realidad, con veces a impiden el desarrollo de los derechos ${ }^{3}$. Nuestro argumento central es que dichas reformas hacen parte de un movimiento más amplio de reestructuración del capital, en lo que respecta a la geopolítica crítica y la nueva división internacional del trabajo, en las sociedades contemporáneas, configurando así una verdadera "ola", en que ha ascendido la agenda neoliberal, con efectos directos en las condiciones de vida de las poblaciones atingidas, en lo particular, en sus formas de trabajo, generando una disminución de su calidad, lo que ha sido identificado por la literatura especializada como "trabajo precario".

Ese ensayo académico se justifica pues el neoliberalismo, con teorías que acercan el sentido común, como la disminución del Estado, así como de los derechos de los trabajadores, para generar empleo, ya empiezan a producir efectos en el mercado laboral de los países donde fueran hechas esas reformas y es importante identificar la eficacia o no de ese argumento, para observar si el argumento generó mejoras sociales a los trabajadores, como más empleo, o si en la verdad el interés económico empresarial he obtenido mejores resultados. Un ejemplo, de esto se refiere al estado penal, cuya función en el contexto contemporáneo es de oprimir y punir de forma cerrada a la pobreza y a los socialmente marginados ${ }^{4}$.

\footnotetext{
${ }^{1}$ HOBSBAWM, 1995.

2 BECK, 2011.

${ }^{3}$ DE SOUZA SANTOS, 2004.

${ }^{4}$ WACQUANT, 2008; 2014; PAULA, 2017.
} estable, en que había seguridad en las relaciones sociales y laborales no es el mismo del mundo contemporáneo ${ }^{1}$. Hoy el elemento central en el mercado laboral es la instabilidad, inseguridad generalizada, que afecta las relaciones entre trabajadores, entre trabajadores y el empresario, los salarios, el medio ambiente laboral, las horas laboradas. La sociedad, la vida y las condutas individuales perdieron sus referencias a la tradición y pasan a ser consideradas como un proyecto, en el sentido experimental, en que cada uno debe buscar la respuesta única para sus cuestiones. No hay referencias anteriores váli- 
Ese debate se hace importante, pues es una discusión, según datos colectados en órganos internacionales, sobre la flexibilización en la legislación laboral que es un discurso muy actual y que no siempre viene con datos, explicaciones y la realidad sobre el mercado laboral después de la aprobación del marco legal. El discurso ganador es que los cambios generan más empleos, posibilidad de que el trabajador tenga más empleos, pueda negociar directamente el contrato laboral y sus derechos con el empleador. Pero en la realidad y después de la aprobación de ese marco legislativo en algunos de esos países esta política pública no obtuvo estos resultados, si no, un aumento del empleo informal, menos horas laboradas, menor salario, por lo tanto, peores condiciones laborales, la llamada precarización laboral y más distante de los derechos humanos y sociales reales ${ }^{5}$ del trabajo decente ${ }^{6}$.

Además de eso, es casi un consenso en la literatura de la sociología de trabajo y de los especialistas en derecho laboral la identificación del mercado laboral con un empeoramiento de las condiciones vividas por los trabajadores. Eso es, todavía, mayor consenso cuando la investigación trata el espacio en la América Latina, inclusive con la creación del concepto de "brasileñización" , del mercado laboral como característica de un trabajo inseguro, insalubre, malo.

Este artículo dedica atención precisamente abordar la cuestión de las malas condiciones laborales o la llamada precarización del trabajo generadas por las leyes de reforma laboral que, intencionalmente o no, generan estos resultados de inestabilidad, inseguridad. Por tanto, la categoría de análisis en ese ensayo, la precarización laboral y su producto el trabajo precario, es una mirada según indicadores de la OIT y el neoliberalismo. Para desarrollo del concepto del se va partir de siete puntos descritos por el sociólogo ${ }^{8}$ Arne Kallenberg, según la Organización Internacional del Trabajo, que tiene la inseguridad como eje central: 1) la incertidumbre en el mercado de trabajo (falta de oportunidades de empleo); 2) la incertidumbre del trabajo (protección inadecuada contra la pérdida de empleo o despido arbitrario); 3) la inseguridad laboral (incapacidad para continuar en una

\footnotetext{
${ }^{5}$ PAULA, 2017.

${ }^{6} \mathrm{GHAl}, 2003$

${ }^{7}$ BECK, 2000

8 KALLENBERG, 2009 y 2011
} 


El término
"precario",
"trabajo precario",
entre otros
sinónimos se
han utilizado
para describir las
transformaciones
que el "mundo del
trabajo" ha estado
sufriendo durante
toda la legislación
mundial, en
contexto reciente,
en países en
desarrollo como
por ejemplo
México y en Brasil,
con una retirada
de las políticas de
Estado y de avance
y agendas de
mercado

El término "precario", "trabajo precario", entre otros sinónimos se para describir las transformaciones que el "mundo del trabajo" ha estado sufriendo durante toda la legislación mundial, en ocupación en particular debido a la falta de límites de artesanía y calificaciones de trabajo); 4) la inseguridad seguridad y salud (malas condiciones de seguridad y salud ocupacional); 5) la experiencia de reproducción de incertidumbre (falta de acceso a la educación básica y la formación profesional);6) la inseguridad de ingresos (ingresos insuficientes; sin pago garantizado o la expectativa de un nivel adecuado de ingresos actuales y futuros); 7) la representación de la inseguridad (falta de derechos individuales en las leyes y los derechos colectivos para negociar) ${ }^{9}$.

La idea, por lo tanto, es presentar un estudio histórico comparado entre Alemania, España, Reino Unido, Italia, y principalmente México y Brasil, para evaluar el efecto de reforma laboral y el cumplimiento del Estado de Bienestar Social, logo, derechos sociales de los trabajadores, teniendo en vista los indicadores de precarización del trabajo de acuerdo con la OIT (Organización Internacional del Trabajo). Dentro de estos hechos sociales que se propone el problema de la investigación: ¿Que tipo de impacto en el mercado laboral ha generado las reformas en las leyes laborales en Alemania, España, Reino Unido, Italia, Brasil y México sobre las condiciones de trabajo? ¿Cuáles fueron los resultados de las reformas laborales en estos países en términos de los criterios de considerados por Kallenberg para la precarización del trabajo?

\section{1. ¿QUÉ ES EL TRABAJO PRECARIO?}

El término "precario", "trabajo precario", entre otros sinónimos se han utilizado para describir las transformaciones que el "mundo del trabajo" ha estado sufriendo durante toda la legislación mundial, en contexto reciente, en países en desarrollo como por ejemplo México y en Brasil, con una retirada de las políticas de Estado y de avance $y$ agendas de mercado ${ }^{10}$.

El término "precariedad", "precarización", "trabajo precario", entre otros sinónimos se han utilizado para describir las transformaciones que el "mundo del trabajo" ha estado sufriendo durante toda la legislación mundial, con una retirada de las políticas de Estado y de avance y agendas de mercado ${ }^{11}$.

\footnotetext{
${ }^{9}$ KALLENBERG, 2009, p. 25 - itálicos añadidos.

10 STANDING, 2014.

${ }^{11}$ STANDING, 2014.
} 
La labor, como precario ha causado inseguridad para los trabajadores, que es un problema también en la concepción subjetiva, es decir, a las relaciones interpersonales, ya sea en el aspecto familiar, o incluso en las relaciones entre los trabajadores mismos no son más de amistad y afinidad en lugar de la competencia, y, además, la relación objetiva con la inestabilidad política establece relación laboral.

Para el sociólogo Kallenberg, el aumento del trabajo precario en el mundo es el resultado de la globalización, la interdependencia económica y la expansión del neoliberalismo. En Brasil y en México el trabajo precario surgirá con el aumento de la privatización, la desregulación del mercado, el avance del neoliberalismo en la década de $1990 .{ }^{12}$

Un de los teóricos que tiene una teoría nueva sobre el trabajo precario es el economista Guy Standing. En su nuevo libro El Precariado: una nueva clase perigosa trata de hablar del trabajo precario, pero más que caracterizarlo, introducir la idea de que es tan grande esa categoría social que ya es una nueva clase social. Según el autor ${ }^{13}$ el concepto marxista del proletariado no es suficiente para contener todas las categorías sociales y las características del trabajo globalizado contemporáneo.

Para el sociólogo André Gorz ${ }^{14}$ los atributos de la clase social marxista: identidad ocupacional, tomada del poder por la revolución, minoría alienada al sistema de empleo, pero con seguridad y bien remunerada, compromiso histórico con la revolución y los cambios. El precariado es una facción social que se diferencia del proletariado pues ese tiene derechos garantizados por leyes, sindicatos que los representan, seguridad y beneficios sociales, son reconocidos por los trabajos o funciones que ejercen; ya el primer, precariado, no trabaja en la función que está capacitado, si tiene capacitación, es una clase políticamente conservadora, no tiene sindicatos que los representa, ni tampoco derechos que les garantizan empleo, despido arbitrario, no tienen horas fijas de trabajo, ni sueldo garantizado, siguen sin identidad ocupacional ${ }^{15}$.

\footnotetext{
12 KALLENBERG, 2009.

13 STANDING, 2015.

14 GORZ, 1995.

15 STANDING, 2015
} 
Los hechos sociales que afectan esa nueva clase social son: 1) flexibilización del trabajo; 2) no hay identidad ocupacional; 3)no pueden realizarse en una carrera profesional; 4) protección laboral insuficiente; 5)derechos sociales y laborales siempre reduciendo o sobre ataques; 6) migración.

En ese punto es que la teoría del precariado de Standing ha recibido muchas críticas, pues él pone la migración como un factor que genera el trabajo precario y el migrante como trabajador precarizado. Pero en el contexto de Europa y Estados Unidos, la cuestión de la migración es muy fuerte y genera impactos en el mercado laboral, pero en el contexto de América Latina, espacio de estudio de la presente tesis, la migración no es un factor tan importante, aunque tengamos un mercado laboral bastante precarizado ${ }^{16}$.

Así que están surgiendo algunos teóricos para tratar una porción de la población que están trabajando o no, más como peores garantías de empleo, trabajo y leyes de seguridad social no les dan estabilidad. A pesar de la diversidad de teorías, que algunos se mostrarán aquí, se pretende una conceptualización del término analíticamente usando los autores Arne Kallenberg (2009) y Erik Olin Wright (2015). Por lo tanto, el trabajo adquiere en la contemporaneidad la característica de la inseguridad para los trabajadores, que es un problema en la concepción subjetiva, es decir, a las relaciones interpersonales, ya sea en el aspecto familiar, o incluso en las relaciones entre los trabajadores mismos no son más de amistad y afinidad en lugar de la competencia, y además, la relación objetiva con la inestabilidad política establecen relación laboral. Arne Kalleberg ${ }^{17}$ pone de manifiesto que la OIT, Organización Internacional del Trabajo, distingue siete cualidades de la precariedad del trabajo, es decir, hay rasgos que caracterizan a una obra tan precaria. Más bien, hay que señalar que el autor identificar la similitud entre la precariedad y la inseguridad, como sinónimos. Las siete características se definen como sigue:

1. la incertidumbre en el mercado de trabajo (falta de oportunidades de empleo)

2. la incertidumbre del trabajo (protección inadecuada contra la pérdida de empleo o despido arbitrario)

${ }^{16}$ WRIGHT, 2015.

17 KALLENBERG, 2009. 


El avanzo de
las fuerzas
conservadoras
en el mundo,
con la creación e
implementación
de la agenda
neoliberal hace
con que las
constituciones
sean más
progresistas y que
detengan una base
de los derechos
mínimos civiles,
políticos
y sociales.

3. La inseguridad laboral (incapacidad para continuar en una ocupación en particular debido a la falta de límites de artesanía y calificaciones de trabajo)

4. la inseguridad seguridad y salud (malas condiciones de seguridad y salud ocupacional)

5. experiencia de reproducción de incertidumbre (falta de acceso a la educación básica y la formación profesional)

6. La inseguridad de ingresos (ingresos insuficientes; sin pago garantizado o la expectativa de un nivel adecuado de ingresos actuales y futuros)

7. la representación de la inseguridad (falta de derechos individuales en las leyes y los derechos colectivos para negociar). ${ }^{18}$

\section{EL CONTEXTO INTERNACIONAL DEL NEOLIBERALISMO Y LAS REFORMAS LABORALES EN ALGUNOS PAÍSES DEL MUNDO}

El mundo antes de los años 90 fue caracterizado como un espacio estable ${ }^{19}$, en que había seguridad en las relaciones sociales y laborales no es el mismo del mundo contemporáneo. Hoy el elemento central en el mercado laboral es la inestabilidad, inseguridad generalizada, que afecta las relaciones entre trabajadores, entre trabajadores y el empresario, los salarios, el medio ambiente laboral, las horas laboradas ${ }^{20}$. Una cuestión está en los derechos garantizados en las constituciones del mundo otro hecho es la efectividad de los derechos (que genera la legitimidad) e otra cuestión es la realidad fática, con veces a impiden el desarrollo de los derechos ${ }^{21}$.

El avanzo de las fuerzas conservadoras en el mundo, con la creación e implementación de la agenda neoliberal hace con que las constituciones sean más progresistas y que detengan una base de los derechos mínimos civiles, políticos y sociales ${ }^{22}$. Así es que las agendas sociales contemporáneas dejan de ser un cambio estructural en la sociedad para buscar apenas la efectividad de algunos derechos y de constitución, incluso siendo esa una crítica a los partidos de izquierda del mundo ${ }^{23}$.

\footnotetext{
18 KALLENBERG, 2009, p. 25.

19 BAUMAN, 2004; BECK, 2000.

20 MASCARO, 2015.

${ }^{21}$ MASCARO, 2015.

22 MASCARO, 2015.

${ }^{23}$ SANTOS, 2004.
} 


\section{CUADRO 1: REFORMAS LABORALES Y SUS EFECTOS: ALEMANIA}

\section{Cambios de la Reforma Laboral}

El caso de la Alemania, que empezó con las reformas laborales en los años 2000 las principales alteraciones que cambiarán el mercado laboral de allá fueran: posibilidad de contratación temporaria de empleados, la contratación puede ser por hasta 18 meses, en cualquier trabajo o sector, exenciones fiscales para los trabajadores que tiene baja renta los beneficios sociales perdieran fuerza y relevancia, con dificultades para alcanzarlos, necesidad del desempleados acepte el primero empleo propuesto (independiente de salario, condiciones, horas, funciones y capacitación) para salir del sistema de apoyo del gobierno, además de disminuir en tiempo del seguro desempleo ${ }^{24}$.

\section{Efectos de la Reforma Laboral}

Los resultados de la reforma laboral en la Alemania fueran la disminución del desempleo, pero con $60 \%$ de los empleos criados en los años 2000 hasta 2015 como temporarios, con plazo fijo, 20 horas semanales ${ }^{25}$; otro punto que contribuyo para aumento del empleo fue los contratos laborales de tiempo parcial con una tasa de 3,4 millones entre los años 2000 y 2015, además de eso $80 \%$ de estos empleos fue para las mujeres ${ }^{26}$.

Según Jaehling ${ }^{27}$ no hay dudas de que las reformas laborales disminuirán la protección legal de los trabajadores, cambiando el mercado laboral para que los trabajadores a aceptar cualquier empleo, incluso los más precarizados, temporarios, parcial, bajos salarios, por la inseguridad del mercado y de la inexistencia de protección social del Estado.

En la investigación de Jaehrling ${ }^{28}$ se afirma que el mercado laboral alemán podría tener desarrollado más y más fuerte si los salarios tuvieran crecido junto con la productividad y la inflación.

\footnotetext{
${ }^{24}$ JAEHRLING, 2017.

25 JAEHRLING, 2017.

${ }^{26}$ JAEHRLING, 2017

27 JAEHRLING, 2017

28 JAEHRLING, 2017
} 


\section{ESPAÑA}

\section{Cambios de la Reforma Laboral}

En la España las leyes de reforma laboral ya empezaran desde los años 80 , con más de 50 cambios legislativos ${ }^{29}$.

En crisis de 2008 todavía ha habido más reformas en el mercado laboral español, como: arreglo para facilitar los cambios en el contrato laboral (jornada, salario, pagamento, funciones, movilidad), facilitar la despedida por motivaciones económicas de la empresa, disminución de la indemnización por la despedida sin justificación, aumento de las horas laborales en los contratos de tiempos parciales, facilidad para despedidas colectivas de trabajadores, congelamiento de los salarios mínimos ${ }^{30}$

\section{Efectos de la Reforma Laboral}

Bustillo y Pinto ${ }^{31}$ hicieran estudio reciente del mercado laboral en España y concluyeran que los contratos temporarios son de corta duración, por ejemplo, en 2014, 25\% de los contratos tienen siete días de duración, apenas $0,4 \%$ con tiempo mayor que un año. Otro punto que afirman es el aumento del trabajo autónomo, o sea, la contratación por el empleador del empleado como si fuera empresa, prestador de servicio autónomo, pero $13 \%$ de ellos trabajan para un único empresario ${ }^{32}$; además, los trabajos del tiempo parcial crecieron de $11 \%$ en 2008 para $16 \%$ en $2015^{33}$

Después de la crisis de 2008, las junciones de dos factores fueron muy importantes para el aumento del poder de negociación de los empresarios, cuáles sean: desempleo masivo y menor oferta de beneficios sociales por el Estado. Así es que todavía con una recuperación del nivel del empleo en España, eso hubo por razón del aumento ${ }^{34}$ de un trabajo precario. Ese impacto fue tan fuerte que las organizaciones internacionales como la OCDE y el Banco Central Europea clamaran por un aumento o políticas de valoración ${ }^{35}$ del salario para mejorar la economía española.

\footnotetext{
${ }^{29}$ SALAS Y PERNÍAS, 2017.

${ }^{30}$ BUSTILLOS E PINTO, 2016.

${ }^{31}$ BUSTILLOS E PINTO, 2016.

32 BUSTILLOS E ESTEVE, 2017.

33 BUSTILLOS E ESTEVE, 2017.

${ }^{34}$ SALAS Y PERNÍAS, 2017.

${ }^{35}$ BUSTILLO E ESTEVE, 2017.
} 


\section{REINO UNIDO}

\section{Cambios de la Reforma Laboral}

En El Reino Unido, las reformas laborales fueran más fuertes y es reconocido como el mercado laboral más flexible y liberal de la EE. UU. ${ }^{36}$. Tiene dos características que la diferencia de los otros países, que son la baja protección al empleo o trabajador y la influencia del mercado para regular las relaciones laborales ${ }^{37}$. Históricamente, las reformas empezadas en los años 80 por Margaret Thatcher trataran de reducir el poder de los sindicatos en los espacios del trabajo y en el mercado laboral, disminuir el empleo público y disminuir la protección del empleado, en el seguro social. ${ }^{38}$

\section{Efectos de la Reforma Laboral}

Según las investigaciones de Grimshaw et $a^{\beta 9}$ los empleos de bajos y medianos salarios no tienen relación o conexión con el costo de la vida, empeorando las cuestiones y índices de pobreza; otra cuestión del empleo en Reino Unido es el empeoramiento de las condiciones laborales de los trabajadores, los empleos del tiempo integral disminuirán, los derechos de los trabajadores, la remuneración, con aumento del empleo temporario.

En resumen, la tasa que se concluye del mercado laboral en el Reino Unido son 2,07 millones de empleos creados en los años de 20082017 , casi $50 \%$ son los autónomos y casi $30 \%$ son empleos temporales, así es que $80 \%$ de los empleos que surgieron en estos 9 años están en una forma atípica de contratación, sin contratos, sin seguridad, sin beneficios sociales, sin las garantías legales criados no período son formas de empleo atípicas. ${ }^{40}$

\footnotetext{
${ }^{36}$ BARBIERE Y CUTULI, 2015.

37 BARBIERE Y CUTULI, 2015.

38 ADISSON Y SIEBERT, 2000.

${ }^{39}$ GRIMSHAW et al, 2017.

${ }^{40}$ GRIMSHAW et al, 2017.
} 


\section{ITALIA}

\section{Cambios de la Reforma Laboral}

En Italia hay una fuerte crisis de la falta del empleo, con disminución del potencial productivo y producción que empeoran ${ }^{41}$ con la crisis de 2008. De 2009 hasta 2015 la tasa de desempleo subió de 6,7\% para $11,9 \%$ y entre los jóvenes de $21,2 \%$ para $40,3 \%{ }^{42}$. Algunas de las medidas de la reforma laboral en Italia, en 2014, para disminuir el desempleo fue: posibilidad de voucher como pagamento de salario (es una forma de pagamento que no tiene contribuciones sociales y impuestos involucrados, disminuir la protección al trabajador), contratos laborales más flexibles a despedidas, contratos laborales de tiempo parcial y temporales ${ }^{43}$.

\section{Efectos de la Reforma Laboral}

La conclusión a que se llegan los autores Fana, Guarascio y Cirillo ${ }^{44}$ es que el mercado laboral también en Italia tuvo un empeoramiento de las condiciones laborales, pasando la inseguridad del mercado y el riesgo de la empresa para los trabajadores, que reciben sin garantías de las contribuciones sociales, con contratos flexibles y trabajos temporales y parciales sin un aumento de la tasa de desempleo.

\section{BRASIL}

\section{Cambios de la Reforma Laboral}

En Brasil la Ley 13.467/17 de Reforma Laboral que empezó la vigencia en noviembre de 2017 todavía sigue con poco tiempo para analice de sus efectos, pero en Ministerio del Trabajo y la institución CAGED, Cadastro Geral de Empregados e Desempregos, divulgaran una investigación con algunos de los impactos de la nueva ley en el mercado Laboral.

\section{Efectos de la Reforma Laboral}

Hubo decrecemos de 12.292 vacancias de trabajo formal, con una tasa de 1.124.090 demisiones en noviembre, según el velatorio una retracción del empleo formal ${ }^{45}$. El cambio se presentó con algunas

\footnotetext{
${ }^{41}$ FANA, GUARASCIO, CIRILLO, 2017.

${ }^{42}$ FANA, GUARASCIO, CIRILLO, 2017.

${ }^{43}$ FANA, GUARASCIO, CIRILLO, 2017.

${ }^{44}$ FANA, GUARASCIO, CIRILLO, 2017.

${ }^{45}$ BRASIL, 2017.
} 
categorías de la ley nueva, como la posibilidad de contratación del empleado por tempo parcial o temporario, teniendo fuerte influencia el empleo en comercio por razones de las fiestas de final del año ${ }^{46}$.

En el trabajo de tempo parcial el sector de comercio tuvo una participación de $55 \%$, después el sector de servicio con $37 \%{ }^{47}$. Analizando el perfil de los trabajadores tenemos 57\% de mujeres de até 29 años, graduadas $(66 \%)^{48}$. O sea, la modalidad de tiempo parcial, con menores salarios mensuales sin beneficios sociales son cumplidas por las mujeres jóvenes y cualificadas.

Desde noviembre de 2017 que, en los clasificados de periódicos grandes corporativos de empresarios como redes de supermercados internacionales, de fast food, divulgaran plazas de empleo temporario con salarios bien abajo del sueldo mínimo en el país semanales ${ }^{49}$. El salario mínimo actual en Brasil es de $\mathrm{R} \$ 937,00$ reales o U\$ 291,94 dólares, por 8 horas laborales o 44 semanales. Hay empresas ofreciendo salarios de $\mathrm{R} \$ 115$ reales por 4 horas laboradas, U\$ 35,00 dólares ${ }^{50}$.

Así es que aun con poco tiempo de la reforma laboral, los impactos el mercado laboral empiezan, con disminución de los salarios en 8.34 veces, contrataciones con jornadas laborales reducidas, sin los beneficios sociales como la seguridad social.

\section{MÉXICO}

\section{Cambios de la Reforma Laboral}

En México, así como en toda América Latina, han sufrido cambios en las leyes desde del avance neoliberal de los años 90, pero la reforma de 2012 fue una de las grandes transformaciones que impactaran fuerte en el mercado laboral| ${ }^{51}$.

Hay más de 38 cambios en la Ley Federal del Trabajo, pero los puntos más relevantes son: la posibilidad de contratación sazonal, temporal, outsourcing, parcial; antigüedad del empleado pasa a no ser relevante para mantener la contratación; la posibilidad del trabaja-

\footnotetext{
${ }^{46}$ BRASIL, 2017.

47 BRASIL, 2017.

48 BRASIL, 2017.

${ }^{49}$ RAMOS, 2017.

50 RAMOS, 2017.

${ }^{51}$ RAMOS, 2017.
} 
dor tener más tareas do que sola la prevista en su función o contrato laboral; tercerizaciones o "cuaterizaciones" (outsourcing); salario por hora; los salarios atrasados solo serán pagado por un plazo máximo de doce meses $^{52}$.

Esos cambios tienen como objetivo no la mejora del empleo o de las condiciones laborales para el trabajador, pero la flexibilidad y más beneficios al empleador ${ }^{53}$.

\section{Efectos de la Reforma Laboral}

Según datos de la Comisión Económica para la América Latina ${ }^{54}(2016)$ presentan que la riqueza en el México aumentó 7,9\% de 2004 hasta 2014, pero la economía creció 2,6\%, además de eso, el $10 \%$ de las familias mexicanas más ricas representa $2 / 3$ de los activos financieros ${ }^{55}$

Otro dato importante de la economía y desarrollo del empleo en México es que después de la reforma laboral de 2012, 51\% de los empleos generados en el mercado laboral fueran por microempresas, con menos de diez empleados, siendo que, en $2016,82,2 \%{ }^{56} \mathrm{de}$ los empleados en esas empresas no tenían contrato laboral.

El mercado laboral mexicano convive con el problema grande de la deterioración de la renta del trabajador, que de 2005 era de U\$ 479 dólares, cambiando en 2016 para U\$ 262 dólares ${ }^{57}$

Es muy importante analizar además de los datos del empleo y desempleo, o sea, si aumentarán o disminuirán con la reforma de 2012, pero también es importante analizar qué tipo, cualificaciones, funciones, horas, tienen eses empleos generados, pues al que parece, aunque la tasa del desempleo disminuyó un poco, las características del empleo creado no fueran las mejores. Es decir, que generarán una masa de trabajadores empobrecidos malas condiciones laborales, que, aunque trabajen, no salen de la condición de pobreza.

Fuente: elaboración propia según datos de diversos artículos ${ }^{58}$.

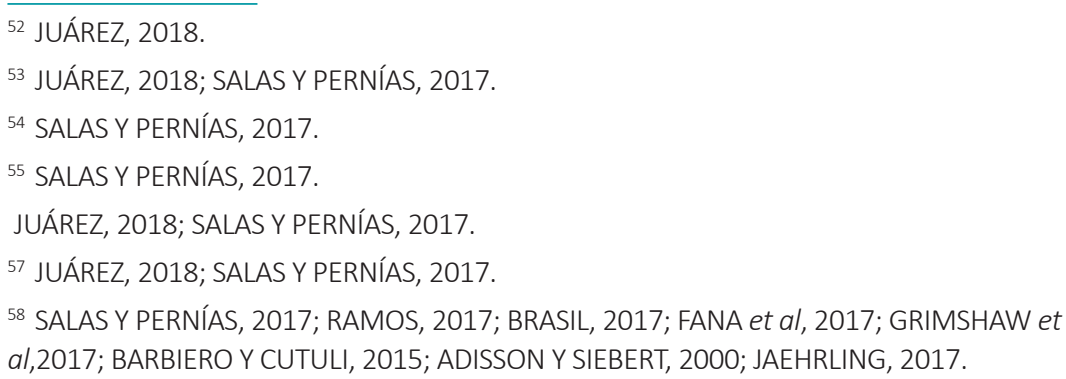




\section{LA REFORMA LABORAL EN EL MUNDO: CASO MÉXICO Y BRASIL}

\subsection{El contexto mundial}

Más recientemente, en 2017 fue divulgado el "Relatorio Global sobre Salarios" pela OIT, Organización Internacional del Trabajo, que afirma que desde 2012 los crecimientos de los salarios reales a nivel global, sacando China que tuve aumento, tuvieran una disminución porcentual de 1,6\% en 2012 para 0,9\% en 2015 del valor.

En 2015 los salarios reales cayeran cerca de 1,3 por ciento em la América Latina y Caribe (sobre todo debido a la baja de los salarios en Brasil), y 5,2 por ciento en la Europa de Leste (debido a la baja de los salarios en la Federación Rusia y Ucrania) $)^{59}$

Pero en los países desarrolladas, del G20, por ejemplo, los salarios tuvieron un aumento, pasarán de los 0,2\% en 2012 para 1,7\% en 2015, la tasa más elevada de los últimos 10 años. En el año de 2015 en los Estados Unidos el crecimiento real de los salarios tubo un aumento de $2,1 \% ; 1,5 \%$ en la Europa del Norte, del Sur, y $1,9 \%$ en los países de la Unión Europea (EE. UU.). ¿Pero, por qué estos resultados? Según la OIT (2017),

Este hecho debe probablemente a una combinación de factores incluido la globalizada, competencias orientadas para a tecnología, la debilidad de las instituciones de mercado del trabajo, y la creciente presión dos mercados financieros para transferir para os investiduras los excedentes generados por grandes empresas ${ }^{60}$

De acuerdo con el estudio la desigualdad salarial es mayor entre los sueldos más altos. Por ejemplo, en la Europa los $10 \%$ que reciben los más altos salarios, obtienen el mismo que $25,5 \%$ de los salarios de todos los trabajadores ${ }^{61}$. Eso cambia y agrava en países en desarrollo como en Brasil que la diferencia está en 35\%, India con $42,7 \%$ y África del Sur con 49,2\% (OIT, 2017).

Otro dato interesante de la investigación es acerca de la diferencia salarial entre hombres y mujeres que se acentúa con la mejor calificación y los mejores puestos de trabajo, es decir, cuanto más elevada el estatus profesional, las funciones, más topo de la

\footnotetext{
${ }^{59}$ OIT, 2017, p.1, traducción libre.

${ }^{60}$ OIT, 2017, p. 2.

${ }^{61}$ OIT, 2017.
} 
La estagnación de los salarios por la cuestión social, la diferenciación entre el crecimiento económico y el no crecimiento de los salarios, implica en una insatisfacción de la sociedad, pues el trabajador no percibe mejoras en su vida y de su familia. pirámide del empleo, más hay diferencia salarial entre hombres y mujeres (OIT, 2017).

Más una conclusión que alcanza el trabajo es que la cuestión del salario es fundamental para una integración económica y también social (OIT, 2017). La estagnación de los salarios por la cuestión social, la diferenciación entre el crecimiento económico y el no crecimiento de los salarios, implica en una insatisfacción de la sociedad, pues el trabajador no percibe mejoras en su vida y de su familia (OIT, 2017). Además de eso, en la cuestión económica, no aumentar los salarios es decir que los trabajadores van a consumir menos, disminuir el crecimiento económico (OIT, 2017).

El informe se concluye con apuntes acerca de políticas públicas para intentar disminuir las diferencias salariales entre los trabajadores de funciones, género, etnia, capacidad distintos, pero sobre todo la idea es central en la importancia de la regulación estatal además de que los países observen también las regulaciones y propuestas hechas por la OIT en las temáticas laborales y de disminución de la desigualdad.

\subsection{El caso de Brasil}

El discurso neoliberal de flexibilidad laboral en Brasil has estado presente desde los años 90 con el presidente Fernando Collor, Itamar Franco y Fernando Henrique Cardoso. Algunas reformas, sobre todo, la Reforma Laboral no habían tomado fuerza, pues la movilizaciones y manifestaciones populares trataban de bloquear cambios normativos que miraban hacia a la "flexibilidad" laboral. Pero a partir de 2015 con una serie de crisis económicas y políticas, así como un cambio de gobierno y agenda política, con la salida de la presidenta electa Dilma Rousseff para el presidente Michel Temer, el contexto social de crisis favoreció la aprobación de la Reforma Laboral en $2017^{62}$.

La ley cambió hechos importantes en el mercado laboral brasileño, que ya es reconocido como precarizado ${ }^{63}$, con la posibilidad de que la negociación colectiva tenga que ser aplicada en los juzgados,

62 TEIXEIRA et al, 2017.

${ }^{63}$ BECK, 2000. 
aunque no este de acuerdo con los derechos constitucionales, humanos y laborales, también contratación de trabajadores autónomos (como si fuera prestaciones de servicios, pero exclusivos del empleador), posibilitando tercerizaciones y fragilizando conceptos legales, los cuales dificulta la actuación de los inspectores, fiscalía del trabajo ${ }^{64}$.

Sobre esta reforma laboral hecha en Brasil, aprobada en julio de 2017, y la cuestión de los trabajadores subcontratados y sus salarios, hay un reciente estudio de economistas Stein et al (2017) sobre las subcontrataciones (autorizadas de manera general para cualquier profesión, a partir de la reforma de Julio) y sus ganancias financieros o sueldos. Antes de la reforma solo se podría hacer la tercerización u outsourcing en casos que la función desempeñada no fuera la finalidad de la empresa o también en los casos de limpieza, conservación y vigilancia ${ }^{65}$

Como todavía la tercerización ${ }^{66} \mathrm{u}$ outsourcing en Brasil solo era posible en los casos descritos arriba, la investigación empírica de Stein et $a /^{67}$ se basa en esas profesiones o funciones. Es importante decir que en los dados encontrados en la investigación ${ }^{68}$ (sacados del RAIS-MTE, en los años de 2007-2014), hecha por Stein et al (2017), se observa que las mujeres están ocupando más los empleos tercerizados; aún, los blancos ocupan más los empleos propios; los empleos tercerizados tienen una mano de obra en

\footnotetext{
$\overline{64}$ TEIXEIRA et al, 2017.

65 STEIN et al, 2017.

66 "Inicialmente, notamos que os trabalhadores terceirizados ganham, em média, menos do que os trabalhadores próprios. As demais estatísticas mostram, porém, que a comparação dos salários médios desses dois grupos de empregados deve ser feita com cautela, uma vez que há diferenças nos valores médios de variáveis importantes para a determinação da remuneração do trabalhador. Por exemplo, os terceirizados são, em média, três anos mais novos e ficam menos tempo no emprego. Há também diferenças significativas na proporção de mulheres e de trabalhadores brancos entre os grupos" (Stein et al, 2017, p.3).

67 STEIN et al, 2017.

68 "O maior diferencial nos salários foi encontrado para os trabalhadores de Telemarketing que transitam entre as duas formas de contratação com-9\% no salário mensal. Trabalhadores de Limpeza e conservação, Montagem e manutenção de equipamentos e TI também apresentam um diferencial negativo, todos entre- $5,5 \%$ e- $6 \%$. Já os trabalhadores envolvidos em atividades de P\&D tem um diferencial médio próximo a-2,5\%. Por outro lado, os trabalhadores das atividades de Segurança/vigilância recebem, em média, 4,9\% a mais quando são terceirizados. O exercício com as ocupações evidencia que existe uma considerável heterogeneidade no diferencial de salário entre empregados terceirizados e próprios conforme as ocupações consideradas. O efeito médio, incondicional a ocupações, é negativo e relativamente pequeno, mas dentro dele encontram-se desde efeitos negativos relativamente mais altos (Telemarketing) até efeitos positivos, como no caso das ocupações de Segurança/Vigilância" (STEIN et al, 2017, p.5)
} 
media 3 años más joven además de no quedaren mucho tiempo en el empleo.

Según el Instituto Brasileño de Geografía y Estadística, un año después de la aprobación de la subcontratación, hay $22 \%$ de los trabajadores formales ${ }^{69}$ bajo esa condición laboral.

El argumento para aprobación de la subcontratación en Brasil es de que existe en la realidad y para dar seguridad jurídica para el trabajador es importante los derechos expresos en ley. Pero eso es una gran falacia, pues se en la sociedad se busca mejorar las condiciones laborales, proporcionar una vida digna a los trabajadores, de acuerdo con normas nacionales e internacionales de derechos humanos, no es razonable que se haga legal algo que no genera salario, jornada laboral, prestaciones, condiciones de salud y higiene laboral, dignos, o sea, el trabajo decente.

\subsection{El caso de México}

En la constitución de México de $1917^{70}$, hay expresas garantías al trabajo digno y decente, como un objetivo del Estado, garantizar el trabajo humanizado y para el crecimiento del individuo y de la economía.

En México, así como en toda América Latina, ha sufrido cambios en las leyes desde del avance neoliberal de los años $90^{71}$, pero la reforma de 2012 fue una de las grandes transformaciones que impactan fuerte en el mercado laboral.

Hay más de 38 cambios en la Ley Federal del Trabajo, pero los puntos más relevantes son ${ }^{72}$ : la posibilidad de contratación sazonal, temporal, outsourcing, parcial; antigüedad del empleado pasa a no ser relevante para mantener la contratación; la posibilidad del trabajador tener más tareas do que sola la prevista en su función o contrato laboral; tercerizaciones o "cauterizaciones" (outsourcing); salario por hora; los salarios atrasados solo serán pagado por un plazo máximo de doce meses.

\footnotetext{
${ }^{69}$ Dino. Divulgacao de notícias. Terceirização abrange 22\% da mão de obra formal com boas perspectivas de crescimento. Revista EXAME, São Paulo, 17 jul 2018.

${ }^{70}$ Artículo 123. Toda persona tiene derecho al trabajo digno y socialmente útil; al efecto, se promoverán la creación de empleos y la organización social de trabajo, conforme a la ley

${ }^{71}$ DE SOUZA SANTOS, 2004.

72 JUÁREZ, 2018; SALAS Y PERNÍAS, 2017.
} 
Esos cambios tienen como objetivo no la mejora del empleo o de las condiciones laborales para el trabajador, pero la flexibilidad y más beneficios al empleador.

Según datos de la Comisión Económica para la América Latina (2016) presentan que la riqueza en el México aumentó 7,9\% de 2004 hasta 2014 , pero la economía creció $2,6 \%$, además de eso, el $10 \%$ de las familias mexicanas más ricas representa $2 / 3$ de los activos financieros ${ }^{73}$.

Otro dato importante de la economía y desarrollo del empleo en México es que después de la reforma laboral de 2012, 51\% de los empleos generados en el mercado laboral fueran por microempresas, con menos de diez empleados, siendo que, en 2016, 82,2\% de los empleados en esas empresas no tenían contrato laboral. A su vez, el mercado laboral mexicano convive con el problema grande de la deterioración de la renta del trabajador, que de 2005 era de U\$ 479 dólares, cambiando en 2016 para U\$ 262 dólares.

Es muy importante analizar además de los datos del empleo y desempleo, o sea, si aumentarán o disminuirán con la reforma de 2012, pero también es importante analizar qué tipo, cualificaciones, funciones, horas, tienen eses empleos generados, pues al que parece, aunque la tasa del desempleo disminuyó un poco, las características del empleo ${ }^{74}$ creado no fueran las mejores. Es decir, que generarán una masa de trabajadores empobrecidos malas condiciones laborales, que, aunque trabajen, no salen de la condición de pobreza.

Según un análisis comparada de investigación, hecha para este trabajo científico, de antes y después de la Reforma Laboral de 2012 se puede observar los 22 puntos más importantes de la reforma y que impactaron directa o indirectamente en el mercado laboral mexicano:

1. La cuestión del "trabajo digno y decente" como un concepto y un objetivo a seguir por el Estado Mexicano.

2. Han fortalecido mucho la cuestión de la Conciliación entre las partes, trabajador y empresario, también para disminuir los procesos en la justicia y los tiempos del trámite.

73 SALAS Y PERNÍAS, 2017.

74 JUÁREZ, 2018. 
3. Otras modalidades de contratación de empleado, "a prueba", por capacitación inicial, temporada, por obra, por estacionalidad y garantizar la seguridad social.

4. Otros criterios para ocupar vacantes no solo por la antigüedad como también por capacitación.

5. Define y incluye el concepto de productividad.

6. Creación de comisiones mixtas de capacitación.

7. Posibilidad de pedir las cuentas a sindicatos, sin sanciones a los trabajadores.

8. Elección de la directiva sindical.

9. Publicitar las informaciones de los contratos colectivos y documentos sindicales.

10. Tornar crimen contratar menores para trabajar, tipificación del trabajo infantil.

11. La retirada del artículo que habla de la prohibición del trabajo nocturno para los menores de 14 a 16 años.

12. Licencia del trabajo para los padres, incluso en casos de adopción.

13. Posibilidad de subcontrataciones/tercerizaciones o "outsourcing", que no puede ser de la actividad principal de la empresa.

14. Posibilidad de responsabilizarse al contratante de la subcontratación por los débitos en la legislación laboral si probado la ilegalidad de la contratación.

15. Autorización a la autoridad laboral para restringir el acceso al trabajo cuando exista peligro inminente para la salud y la vida de los trabajadores.

16. Instalaciones laborales de acceso a personas con discapacidad.

17. Las sanciones también fue un punto de cambio, aumentando el valor de la multa para el empresario que no la cumpla de 315 a 5 mil veces el salario mínimo general.

18. Sanciones al acoso u hostigamiento en el trabajo.

19. Medidas protectoras a las mujeres madres trabajadoras.

20. Prohibición de discriminación por género, acoso o hostigamiento, así como pedido de comprobación de embarazo. 
21. Distribución del descanso pre y postnatal y licencia maternidad y paternidad.

22. Protección de derechos laborales a grupos vulnerables como trabajadores de minas, rurales y domésticos.

Aún con todas estés cambios normativos y el objetivo de disminución de las demandas judiciales, por priorizar altas multas por los empresarios no cumplieren la ley o aún por fortalecimiento de la conciliación en la justicia, de 1995 hasta 2015 hubo un aumento de $132 \%{ }^{75}$ en procesos judiciales en pleitos laborales.

Primer sobre la cuestión del desempleo en México, que era uno de los objetivos de la ley, según Rafael Juárez, de diciembre de 2012 a febrero de 2017, se observó la creación de 2559035 nuevos puestos de trabajo afiliados entre IMSS, de los cuales más de $40 \%$ han sido mujeres, 25\% para jóvenes de entre 15 y 29 años, además, $80 \%$ son permanentes ${ }^{76}$.

Sobre las diferentes formas de contratación tratadas por la LFT, de acuerdo con el Módulo de Trayectorias Laborales del INEGI, en 2015 un 6.5\% de los empleos formales estaban contratados bajo la modalidad de periodo de prueba y otro $6.7 \%$ bajo la de capacitación inicial ${ }^{77}$.

En estos puntos es importante una reflexión crítica sobre los empleos, pues, aunque muchos trabajadores salieron del mercado informal para ingresar como trabajadores formalizados, hay que observar bajo cuales condiciones están trabajando, principalmente, si este trabajo es digno y está generando la posibilidad de salida de la pobreza.

Según Rodríguez ${ }^{78}$ la ENOE presenta dados de 2017 que la tasa de informalidad laboral en México ha sabido para 57,3\% de toda la población ocupada en el país. Eso es un dato muy importante, pues es reconocer que más de la mitad de los trabajadores mexicanos no están recibiendo un amparo de la ley de protección con contrato laboral, prestaciones de ley, aguinaldo, vacaciones o amparo de las instituciones laborales.

\footnotetext{
${ }^{75}$ MÉXICO, Junta Local de Conciliación y Arbitraje de la Ciudad de México. Disponible en: $<$ http://www.juntalocal.cdmx.gob.mx/>.

76 JUÁREZ, 2017, p. 61.

77 JUÁREZ, 2017, p.62.

78 RODRÍGUEZ, 2017.
} 
En la temática de los salarios después de la Reforma Laboral de 2012, dice Rodríguez en sus resultados que,

Un ejercicio practicado por el Centro de Análisis Multidisciplinario de la Universidad Nacional Autónoma de México enfocado a examinar el poder adquisitivo del salario en el marco de la política laboral de los últimos años ha establecido que la pérdida de la capacidad de compra de los ingresos laborales en México es de tal magnitud, que en caso de llegar a autorizarse en el futuro incrementos salariales semejantes a los otorgados a partir del 1 de enero de 2017 (3.9\% anual), sería necesario llegar hasta el año de 2044 para que un trabajador, con salario mínimo, logre adquirir una Canasta Alimentaria Recomendable ${ }^{79}$.

Por tanto, el salario mínimo en el actual momento está muy debajo de las condiciones mínimas de dignidad y pobreza. Aún, con la Reforma hubo cambio en el poder de compra del mexicano, bajando el salario mínimo por hora en las estadísticas de OCDE:

En los hechos se dio un abaratamiento de la fuerza de trabajo de tal magnitud que, de acuerdo con el ranking de salarios mínimos por hora de la OCDE, desde 2013 a 2015, México permanece como el país con el salario real más bajo de los países que conforman dicha organización con apenas 1.01 dólares por hora(...) $)^{80}$.

Sobre la subcontratación, esta forma de ya estaba en uso en México, según muchos expertos del tema ${ }^{81}$, pero de manera ilegal. La ley por lo tanto legalizó una práctica del mercado laboral ${ }^{82}$. El punto es que en el discurso oficial los trabajadores estaban trabajando con esa modalidad de contratación y con eso la ley debería apenas legalizarla, así como el discurso para aprobarla en Brasil. Lo que no es la finalidad social de apenas generar trabajo o cualquier tipo de trabajo, si no un trabajo con salario, jornada laboral, prestaciones, condiciones de salud y higiene laboral, dignos, con derechos individuales y colectivos democráticos y de seguridad a los trabajadores, o sea, la promoción del trabajo decente ${ }^{83}$.

\footnotetext{
79 RODRÍGUEZ, 2017, p.5.

80 GAYTÁN, 2016, p. 99.

81 JUÁREZ, 2017; GAYTÁN, 2016; FERNÁNDEZ, 2009.

82 JUÁREZ, 2017.

${ }^{83}$ GHAI, 2003; OIT, 2017.
} 


\section{CONCLUSIÓN}

Considerando el objetivo de este artículo, a saber: analizar los impactos generados en los mercados laborales tras las reformas constitucionales, en general, y la aprobación de leyes laborales "más flexibles", en diferentes países en el mundo (en Alemania, España, Reino Unido, Italia), a partir de la década de los 1990, y, en lo particular, discutiendo en detalle el caso de Brasil y México, se puede considerar que:

1. en cuanto al contexto macro:

La conclusión inicial de esa investigación es que la disminución de la tasa de desempleo no genera la disminución de la pobreza, por el hecho de que las plazas de trabajo no son bien pagadas 0 son de pocas horas laboradas al día, con salarios abajo del mínimo, no más garantizando una vida digna al trabajador.
La conclusión inicial de esa investigación es que la disminución de la tasa de desempleo no genera la disminución de la pobreza, por el hecho de que las plazas de trabajo no son bien pagadas o son de pocas horas laboradas al día, con salarios abajo del mínimo, no más garantizando una vida digna al trabajador.

Los datos nos informan que la idea inicial de las reformas laborales en todos los países analizados era de flexibilidad en la ley, con objetivo de generar más empleo, formalizarlos o aún tornar hechos ilegales, como la subcontratación, un tipo de empleo legal y formal. En Alemania se hubo un aumento en los empleos formales, pero $60 \%$ de ellos son temporales con apenas 20 horas semanales, y $80 \%$ de las mujeres más afectadas por estés empleos precarizados por la inseguridad en la contratación temporal y pocas horas laboradas lo que muchas veces reflejan en pocos ingresos, además del aumento de la desigualdad salarial ${ }^{84}$. En España los empleos de tiempo parcial crecieron, la contratación de trabajadores autónomos, congelamiento de los salarios y la facilidad del despido, son características actuales del mercado laboral español. En Reino Unido, el mercado más flexible, con 2,07 millones de empleos creados en los años de $2008-2017$, casi $50 \%$ son los autónomos y casi $30 \%$ son empleos temporales, así es que $80 \%$ de los empleos que surgieron en estos 9 años están en una forma atípica de contratación, sin contratos, sin seguridad, sin beneficios sociales, sin las garantías legales criados no período son formas de empleo atípicas. En Italia, hubo el aumento de los contratos temporales y flexibles, con bajos ingresos y inseguridad en la contratación. 
Así que en algunos de los países ha generado un aumento en la oferta de empleo, pero con contrataciones atípicas, inseguras, con bajos salarios, sin prestaciones y de tiempo parcial o temporario, o sea, la disminución en la tasa de desempleo no ha generado, en ninguno de los países, una oferta de empleos decente, con condiciones dignas de vida y de consumo, apenas ha ampliado la precarización laboral, sobre todo, en la cuestión de la inseguridad en los derechos, en la contratación y seguridad social, en los ingresos y jornada laboral.

2. en cuanto a los casos de Brasil e México:

Sobre los casos de Brasil y México, los objetivos de las leyes también era disminución de la tasa de desempleo y una fuerza especial para aprobación de la subcontratación que ya existía en estos dos países, pero era una modalidad ilegal de contratación.

Como resultado, se puede observar que no hubo aumento de los salarios para los trabajadores en ninguno de estos dos países, la disminución de la tasa de desempleo fue resultado de aumento de empleos temporales, sin seguridad para una contratación formal, y el aumento significativo de la subcontratación u outsourcing.

La flexibilidad en los contratos laborales no reflejó en creación de trabajas decentes si no apenas en aumento de concentración de riqueza, bajos salarios y contrataciones sin seguridad laboral, o sea, los empleos generados no son capaces de enfrentar la cuestión de disminución de la pobreza, de la desigualdad social y laboral, apenas desarrollaron un mercado laboral, también en México y en Brasil, más precarizado.

Resta decir que esta es una investigación científica aún en analice y en búsqueda de más dados, sobre todo los demás puntos del trabajo precario. Pero, desde de ese momento la idea es empezar a hablar de los cambios en el mercado laboral en los dos países, Brasil y México, en los aspectos de formas de contratación precarizada ( $\sin$ contrato formal de trabajo), la jornada del trabajo, rebaja en los salarios, debilidad sindical y flexibilización en los derechos y en la negociación colectiva. 


\section{REFERÊNCIAS}

RODRIGUEZ, Carlos Acevedo. Precarización laboral y legitimación del modelo económico y político: Iquique-Chile, como caso de estudio. Gaceta Laboral, Maracaibo, v. 17, n. 1, p. 31-61, 2011.

ADISSON, J. T; SIEBERT, W. S. Labor market reform in the United Kingdon: from thatcher to Blair. Journal of private enterprise, 2000 , v. 15, issue spring 2000, 1-34

ANTUNES, R. A nova morfologia do trabalho. Conselho, 2013, 93.

BARBIERI, P.; CUTULI, G. Employment protection legislation, labour market dualism, and inequality in Europe, European sociological Review, 32 (4), 2015, 501- 516.

BARBOSA, Rosangela Nair de Carvalho. As reformas trabalhistas neoliberais na América Latina: o caso do México. Anais do XVI Encontro Nacional de Pesquisadores em Serviço Social 16.1. 2019.

BAUMAN, Zygmunt. Trabajo, consumismo y nuevos pobres. Editorial Gedisa, 2000.

BECK, U. Un nuevo mundo feliz: la precariedad del trabajo en la era de la globalización. Paidós, 2007.

BENSUSÁN, Graciela; ALCALDE, Arturo. El sistema de justicia laboral en México: situación actual y perspectivas. 2013.

BRAGA, Ruy. A Política do precariado: do populismo à hegemonia lulista. São Paulo: Boitempo. 2012.

BUSTILLO, R. M.; PINTO, F. H. Reducing precarious work in Europe through social dialogue: precarious employment in Spain, Brussels. 2016. Disponível em: <http://www.research.mbs.ac.uk/>.

BUSTILLO, R. M; ESTEVE, F. The neverending story. labour market deregulation and the performance of the spanish labour market. In. PIASNA, A; MYANT, M. Myths of employment deregulation: how it neither creates jobs nor reduces labour market segmentation. Brussels. ETUI. 2017.

CARD D.; HEINING J.; KLINE P. Workplace heterogeneity and the rise of german wage inequality, Quarterly journal of economics, 128 (3), 967-101,2016.

SANTOS, Boaventura de Sousa. Reinventar la democracia: reinventar el Estado. Editorial Abya Yala, 2004.

DINO. Divulgação de notícias. Terceirização abrange $22 \%$ da mão de obra formal com boas perspectivas de crescimento. Revista EXAME, São Paulo, 17 jul 2018. 
FANA, M; GUARASCIO, D; CIRILLO, V. The crisis and labour Market reform in Italy: a regional analysis of the job act. In. PIASNA, A; MYANT, M. Myths of employment deregulation: how it neither creates jobs nor reduces labour market segmentation. Brussels. ETUI. 2017

GHAI, D. Trabajo decente. Concepto e indicadores. Revista internacional del trabajo, 122(2), 125-160. 2003.

GRIMSHAW, D; JOHNSON, M; KEIZER, A; RUBERY, J. The governance of employment protection in the UK: how the state and employers are undermining decent standards. In. PIASNA, A; MYANT, M. Myths of employment desregulation: how it neither creates jobs nor reduces labour market segmentation. Brussels. ETUI. 2017.

HOBSBAWN, Eric. Era dos extremos: o breve século XX. Companhia das Letras, 1995.

JAEHRLING, K. The atypical and gendered 'employmet miracle' in Germany: a result of employment protection reforms or long term structural changes? In. PIASNA, A; MYANT, M. Myths of employment deregulation: how it neither creates jobs nor reduces labour market segmentation. Brussels. ETUI. 2017.

KALLEBERG, Arne L. Good jobs, bad jobs: The rise of polarized and precarious employment systems in the United States, 1970s-2000s. Russell Sage Foundation, 2011.

KALLENBERG, Arne. O crescimento do trabalho precário: um desafio global. Rev. bras. Ci. Soc., São Paulo, v. 24, n. 69, p. 21, 2009.

MASCARO, A. L. Estado e forma política. Boitempo Editorial. 2015.

MAY, Tim. Pesquisa social. Questões, métodos e processos, v. 3. 2014.

OIT. El trabajo en el mundo: la situación mundial dei empleo. Genebra. 1994.

OIT. Relatório Global sobre os Salários 2016/17. 2017. Disponible en: <conricyt.mx.ezproxy.conricyt.org/Buscador/Conricyttback/ index.php?c=3\&i=52>.

PAULA, S. C. Entre el Leviatán y la bestia colosal: la violencia estatal en México como fenómeno de la ola punitiva. 1. ed. Buenos Aires: Ciudad Autónoma de Buenos Aires, 2017.

RODRIGUEZ, J.J.G. Reforma laboral: um panorama de 3 años de su promulgación. Câmara de Diputados de México: Centro de Estudios Sociales y de Opinión Pública. 2016.

ROMERO, Luis Quintana; ACEVEDO, Blanca E. Garza. La reforma laboral em México y sua efectos econômicos. Revista do TST, v. 83, no3, Jul/set, São Paulo: Lex Editora, 2017. 
SALAS, C., PERNIAS, Tomas. Experiências internacionais; projeto de pesquisa Subsídios para a discussão sobre a reforma trabalhista no Brasil. Textos de discussão n. 2. Campinas: Unicamp/Cesit, 2017.

SALAS, Carlos. Labour, income and social programmes in contemporary Mexico. In: United Nations Development Programme, Social Protection, Growth and Employment: Evidence from India, Kenya, Malawi, Mexico, Peru and Tajikistan, UNDP, New York, 2013: 201-230.

STANDING, Guy. O precariado e a luta de classes. [Online], 103 | 2014, colocado online no dia 26 maio 2014, criado a 29 maio 2015. URL: <http://rccs.revues.org/5521; DOI : 10.4000/ rccs.5521>.

STANDING, Guy. O precariado: A nova classe perigosa. 1. Ed. Belo Horizonte. Autêntica Editora. 2014.

STANDING, Guy. The Precariat and Class Struggle. RCCS Annual Review [Online], 7 | 2015, Online since 01 October 2015, connection on 29 January 2016. Disponível em: <http://rccsar. revues.org/585>; DOI: 10.4000/rccsar.585.

TEIXEIRA, M. O. et al. Contribuição crítica à reforma trabalhista. Campinas: UNICAMP, 2017.

TEIXEIRA, M. O.; KREIN, J. D.; BIAVASCHI, M. B.; GALVAO, A.; ALMEIDA, P. F.; ANDRADE, H. R. Contribuição crítica à reforma trabalhista. Campinas: UNICAMP, 2017.

WACQUANT, L. J. Punir os pobres: a nova gestão da miséria nos Estados Unidos (V. 6). Freitas Bastos Editora. 2001.

WACQUANT, L. Os subúrbios populares em tempos de marginalidade avançada. Revista PPCAAM Minas, 1(1), 11. 2008. WRIGTH, Erik Olin. Understanding Class (Is the Precariat a Class). Ed. University of Wisconsin. Madison. 2015. 